01

\title{
Влияние столкновений на спектр резонансной флуоресценции трехуровневых атомов c V-конфигурацией уровней
}

\author{
(C) А.И. Пархоменко, А.М. Шалагин \\ Институт автоматики и электрометрии Сибирского отделения РАН, \\ 630090 Новосибирск, Россия \\ e-mail: par@iae.nsk.su, shalagin@iae.nsk.su
}

Поступила в редакцию 21.07.2020 г.

В окончательной редакции 27.12.2020 г.

Принята к публикации 26.05.2021 г.

\begin{abstract}
Теоретически исследовано влияние столкновений на спектр резонансной флуоресценции трехуровневых атомов с V-конфигурацией уровней при возбуждении двумя монохроматическими резонансными полями. Анализ проведен для систем с малым доплеровским уширением по сравнению с частотой столкновений (большие давления буферного газа) и для общего случая произвольного изменения (от полного сбоя до полного сохранения) фазовой памяти на любом из трех переходов в V-системе. Показано, что в трехуровневых атомах V-типа, возбуждаемых двумя монохроматическими полями, спектр резонансной флуоресценции на одном из переходов (количество спектральных линий и их ширины) сильно зависит от присутствия резонансного излучения на смежном переходе. В определенных условиях ширины линий будут полностью определяться релаксационными характеристиками смежного перехода и, в частности, могут быть многократно сужены при росте интенсивности излучения на смежном переходе. Показано, что несмотря на однородный характер уширения линий поглощения, спектр резонансной флуоресценции обладает ярко выраженной анизотропией по отношению к взаимной ориентации волновых векторов спонтанного и возбуждающих излучений. Установлено, что ширины линий в спектре резонансной флуоресценции в определенных условиях пропорциональны коэффициенту диффузии взаимодействующих с излучением атомов. Этот факт может служить основой спектроскопического метода измерения транспортных частот столкновений поглощающих излучение частиц с буферными. Показано, что эффекты фазовой памяти при столкновениях приводят к сильной качественной и количественной модификации спектра резонансной флуоресценции.
\end{abstract}

Ключевые слова: резонансная флуоресценция, трехуровневые атомы, столкновения, когерентность, спектр.

DOI: $10.21883 /$ OS.2021.09.51333.201-21

\section{1. Введение}

Исследованию резонансной флуоресценции, возникающей под действием сильного монохроматического излучения, уделяется большое внимание на протяжении уже многих лет. Важное место в этих исследованиях занимает изучение эффектов изменения ширин спектральных компонент резонансной флуоресценции многоуровневых систем, возбуждаемых лазерными полями. В теоретических работах [1-3] исследован спектр резонансной флуоресценции трехуровневых атомов с Vконфигурацией уровней при возбуждении двумя монохроматическими резонансными полями. В этих работах показано, что ширины и относительные амплитуды спектральных линий флуоресценции зависят от атомных параметров и от относительной интенсивности полей. При большом различии интенсивностей полей ширина линии спонтанного испускания на одном из переходов будет равна ширине линии поглощения на другом смежном переходе. В случае большого различия скоростей спонтанного распада возбужденных состояний линия спонтанного испускания на одном из переходов многократно сужается с ростом интенсивности излучения на смежном переходе. Этот эффект сужения спектральных линий флуоресценции зарегистрирован экспериментально [4].

В работах [2,3] предполагалось, что единственным релаксационным механизмом является спонтанный распад уровней, а движение и столкновения атомов не учитывались. В работе [1] столкновения описывались простейшей моделью релаксационных констант (скорость атома при столкновении не изменяется), когда их влияние сводится к перенормировке радиационных констант релаксации. При этом движение атомов в [1] также не учитывалось. Вместе с тем полный и корректный учет процессов релаксации при расчете спектров резонансной флуоресценции, как известно, имеет принципиальное значение. В настоящей работе теоретически исследован спектр резонансной флуоресценции движущихся трехуровневых атомов с V-конфигурацией уровней, находящихся в поле двух сильных монохроматических волн и испытывающих столкновения с атомами буферного газа.

Анализ проведен в модели сильных столкновений (распределение атомов по скоростям после столкновения не зависит от значения скорости до столкновений) для общего случая произвольного изменения (от полно- 


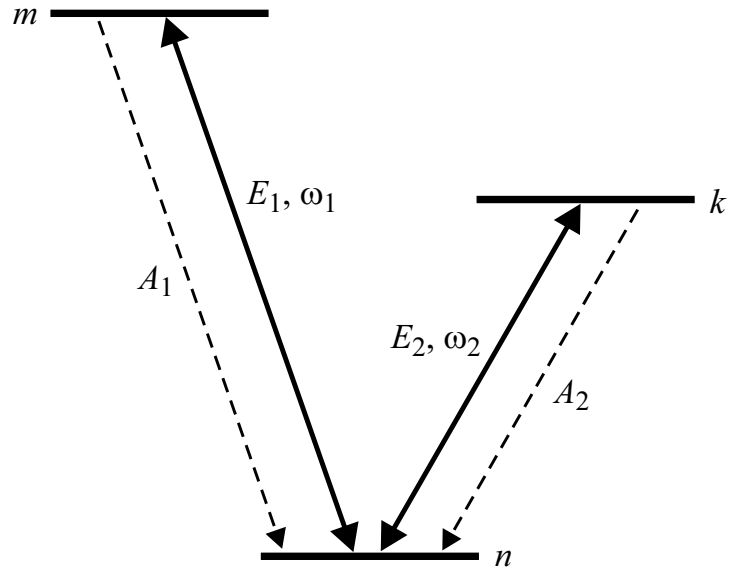

Рис. 1. Схема энергетических уровней и переходов. Сплошными стрелками обозначены переходы под действием излучения, штриховыми - спонтанные радиационные переходы.

го сбоя до полного сохранения) фаз наведенных излучениями дипольных моментов при упругих столкновениях частиц газа. Столкновения, сохраняющие фазу, приводят, как известно, к эффекту Дике сужения спектральных линий $[5,6]$. Рассмотрен случай слабоселективного по скоростям взаимодействия атомов с излучением, соответствующий малому доплеровскому уширению по сравнению с суммой частоты столкновений и скорости радиационной релаксации. Это позволило применить простой метод расчета, основанный на процедуре усреднения по скоростям в самих кинетических уравнениях для матрицы плотности [7]. В итоге получены уравнения, совпадающие с соответствующей системой уравнений для покоящихся частиц с точностью до переопределения констант релаксации, в которых отражено движение частиц по диффузионному закону.

\section{2. Полевое расщепление уровней энергии}

Исследование спектра резонансной флуоресценции состоит в анализе спектра спонтанного испускания с верхнего уровня, возбуждаемого внешним излучением перехода. Прежде чем детально заняться этим исследованием в трехуровневых V-атомах, возбуждаемых двумя монохроматическими полями, установим структуру этого спектра (положение и число спектральных линий) без проведения сложных расчетов, а используя только представление о полевом расщеплении уровней и положении уровней квазиэнергии на основе анализа уравнений для амплитуд вероятностей.

Рассмотрим поведение изолированного трехуровневого атома (схема уровней показана на рис. 1) под действием двух монохроматических полей $\operatorname{Re} \mathbf{E}_{1} \exp \left(-i \omega_{1} t\right)$ и $\operatorname{Re} \mathbf{E}_{2} \exp \left(-i \omega_{2} t\right)$ с частотами $\omega_{1}$ и $\omega_{2}$, близкими к частотам $\omega_{m n}$ и $\omega_{k n}$ переходов $m-n$ и $k-n$. Релаксационные процессы не учитываем. Представим волновую функцию атома, находящегося во внешних полях, в виде линейной комбинации волновых функций стационарных состояний

$$
\Psi(t)=\sum_{j=n, m, k} a_{j}(t) \psi_{j} e^{-i E_{j} t / \hbar},
$$

где $\psi_{j}$ - координатная часть волновой функции стационарного состояния $j, E_{j}-$ энергия атома в $j$-м стационарном состоянии. Для амплитуд вероятностей $a_{j}(t)$ состояний $j$ из уравнения Шредингера следует система уравнений

$$
\begin{gathered}
\frac{d}{d t} a_{n}=i G_{1}^{*} e^{i \Omega_{1} t} a_{m}+i G_{2}^{*} e^{i \Omega_{2} t} a_{k}, \\
\frac{d}{d t} a_{m}=i G_{1} e^{-i \Omega_{1} t} a_{n}, \\
\frac{d}{d t} a_{k}=i G_{2} e^{-i \Omega_{2} t} a_{n},
\end{gathered}
$$

где

$$
\begin{array}{cc}
G_{1}=\frac{d_{m n} E_{1}}{2 \hbar}, & G_{2}=\frac{d_{k n} E_{2}}{2 \hbar}, \\
\Omega_{1}=\omega_{1}-\omega_{m n}, & \Omega_{2}=\omega_{2}-\omega_{k n},
\end{array}
$$

$d_{m n}$ и $d_{k n}$ - матричные элементы дипольного момента переходов $m-n$ и $k-n$. Уравнения (2) представляют собой однородную систему линейных дифференциальных уравнений с коэффициентами, зависящими от времени. Сделаем следующую подстановку:

$$
a_{n}=\widetilde{a}_{n}, \quad a_{m}=\widetilde{a}_{m} e^{-i \Omega_{1} t}, \quad a_{k}=\widetilde{a}_{k} e^{-i \Omega_{2} t},
$$

с помощью которой уравнения (2) приходят к более простому виду

$$
\begin{aligned}
& \frac{d}{d t} \widetilde{a}_{n}=i G_{1}^{*} \widetilde{a}_{m}+i G_{2}^{*} \widetilde{a}_{k}, \\
& \frac{d}{d t} \widetilde{a}_{m}=i \Omega_{1} \widetilde{a}_{m}+i G_{1} \widetilde{a}_{n}, \\
& \frac{d}{d t} \widetilde{a}_{k}=i \Omega_{2} \widetilde{a}_{k}+i G_{2} \widetilde{a}_{n},
\end{aligned}
$$

где коэффициенты уже не зависят от времени. Решение дифференциальных уравнений (5) имеет вид

$$
\begin{gathered}
\tilde{a}_{j}=b_{j 1} e^{i \alpha_{1} t}+b_{j 2} e^{i \alpha_{2} t}+b_{j 3} e^{i \alpha_{3} t}, \\
j=n, m, k,
\end{gathered}
$$

где $\alpha_{1}, \alpha_{2}, \alpha_{3}$ - корни характеристического уравнения

$$
\begin{aligned}
& \alpha\left(\alpha-\Omega_{1}\right)\left(\alpha-\Omega_{2}\right)-\left|G_{1}\right|^{2}\left(\alpha-\Omega_{2}\right) \\
& -\left|G_{2}\right|^{2}\left(\alpha-\Omega_{1}\right)=0 .
\end{aligned}
$$

После подстановки функций (6) в систему дифференциальных уравнений (5) получаем линейную однородную систему алгебраических уравнений для коэффициентов $b_{j s}(s=1,2,3)$ :

$$
\alpha_{s} b_{n s}-G_{1}^{*} b_{m s}-G_{2}^{*} b_{k s}=0,
$$




$$
\begin{aligned}
& \left(\alpha_{s}-\Omega_{1}\right) b_{m s}-G_{1} b_{n s}=0, \\
& \left(\alpha_{s}-\Omega_{2}\right) b_{k s}-G_{2} b_{n s}=0 .
\end{aligned}
$$

Подставим выражения (6), (4) в формулу (1) и получим общее решение для волновой функции в виде

$$
\begin{aligned}
& \Psi(t)=\psi_{n} e^{-i E_{n} t / \hbar}\left[b_{n 1} e^{i \alpha_{1} t}+b_{n 2} e^{i \alpha_{2} t}+b_{n 3} e^{i \alpha_{3} t}\right] \\
& +\psi_{m} e^{-i E_{m} t / \hbar}\left[b_{m 1} e^{i \alpha_{1} t}+b_{m 2} e^{i \alpha_{2} t}+b_{m 3} e^{i \alpha_{3} t}\right] e^{-i \Omega_{1} t} \\
& +\psi_{k} e^{-i E_{k} t / \hbar}\left[b_{k 1} e^{i \alpha_{1} t}+b_{k 2} e^{i \alpha_{2} t}+b_{k 3} e^{i \alpha_{3} t}\right] e^{-i \Omega_{2} t} .
\end{aligned}
$$

Из формулы (9) видно, что каждый из невозмущенных уровней $n, m, k$ под действием полей излучения расщепляется на три уровня квазиэнергии:

$$
\begin{gathered}
E_{n 1}=E_{n}-\hbar \alpha_{1}^{\prime}, \quad E_{n 2}=E_{n}-\hbar \alpha_{2}^{\prime}, \\
E_{n 3}=E_{n}-\hbar \alpha_{3}^{\prime}, \quad E_{m 1}=E_{m}-\hbar\left(\alpha_{1}^{\prime}-\Omega_{1}\right), \\
E_{m 2}=E_{m}-\hbar\left(\alpha_{2}^{\prime}-\Omega_{1}\right), \quad E_{m 3}=E_{m}-\hbar\left(\alpha_{3}^{\prime}-\Omega_{1}\right), \\
E_{k 1}=E_{k}-\hbar\left(\alpha_{1}^{\prime}-\Omega_{2}\right), \quad E_{k 2}=E_{k}-\hbar\left(\alpha_{2}^{\prime}-\Omega_{2}\right), \\
E_{k 3}=E_{k}-\hbar\left(\alpha_{3}^{\prime}-\Omega_{2}\right),
\end{gathered}
$$

где $\alpha_{s}^{\prime}-$ вещественная часть величины $\alpha_{s}$. Значения квазиэнергий подуровней зависят от $\left|G_{1}\right|,\left|G_{2}\right|, \Omega_{1}, \Omega_{2}$. Частоты переходов между уровнями квазиэнергии состояния $n$ и, например, состояния $m$ определяют положения центров линий резонансной флуоресценции на переходе $m-n$ (рис. 2). Из выражений (10) следует, что в общем случае при произвольных значениях интенсивностей и отстроек частот излучений спектр резонансной флуоресценции может содержать до семи линий.

В случае точного резонанса для сильных полей $\left(\Omega_{1}=\Omega_{2}=0\right)$ характеристическое уравнение (7) имеет корни $\alpha_{1}=0, \alpha_{2,3}= \pm G$, где $G=\sqrt{\left|G_{1}\right|^{2}+\left|G_{2}\right|^{2}}$. При этом, как следует из выражений (10), спектр резонансной флуоресценции на каждом переходе состоит из пяти линий. На переходе $m-n$ спектральные компоненты расположены в окрестностях частот $\omega_{m n}, \omega_{m n} \pm G$, $\omega_{m n} \pm 2 G$.

Проведенный выше анализ дал нам важную физическую информацию о количестве линий в спектре резонансной флуоресценции и об их расположении в шкале частот. В частности, можно отметить, что в отличие от спектра резонансной флуоресценции в двухуровневой системе, представленном триплетом Моллоу [8], в рассмотренном случае число компонент спектра достигает семи. Решение другого важного вопроса, касающегося относительных амплитуд и ширин компонент спектра, можно получить лишь при учете процессов релаксации, что в полной мере можно сделать только в аппарате матрицы плотности.

\section{3. Исходные уравнения}

Рассмотрим газ трехуровневых поглощающих частиц, находящихся в смеси с буферным газом. Столкновениями между поглощающими частицами пренебрежем, полагая концентрацию буферного газа много большей концентрации поглощающего газа. Пусть на поглощающие частицы, схема уровней которых показана на рис. 1, воздействуют два монохроматических поля: $\operatorname{Re} \mathbf{E}_{1} \exp \left(i \mathbf{k}_{1} \mathbf{r}-i \omega_{1} t\right)$ и $\operatorname{Re} \mathbf{E}_{2} \exp \left(i \mathbf{k}_{2} \mathbf{r}-i \omega_{2} t\right)$ с частотами $\omega_{1}$ и $\omega_{2}$, близкими к частотам $\omega_{m n}$ и $\omega_{k n}$ переходов $m-n$ и $k-n$ (здесь $\mathbf{E}_{1}, \mathbf{E}_{2}$ и $\mathbf{k}_{1}, \mathbf{k}_{2}-$ напряженности электрических полей и волновые вектора излучений). Спонтанные распады уровней $m$ и $k$ по каналам $m \rightarrow n$ и $k \rightarrow n$ характеризуются константами $A_{1}$ и $A_{2}$. Считаем, что нижний уровень $n$ принадлежит основному состоянию, т.е. не релаксирует.

В трехуровневых V-атомах, возбуждаемых двумя монохроматическими полями, спонтанное испускание происходит на двух переходах: $m-n$ и $k-n$. Не уменьшая общности решаемой задачи, мы ограничимся изучением спектра спонтанного испускания только на одном переходе $m-n$. Спонтанное испускание на переходе $m-n$ будем описывать, согласно рецепту [5,9], с помощью введения эффективного классического поля $E_{\mu}$. При таком подходе вычисление спектра спонтанного испускания формально аналогично определению работы классического пробного поля $E_{\mu}$, если в последней задаче выделить только испускание и под $E_{\mu}$ понимать амплитуду нулевых колебаний вакуума, отвечающую узкому спектральному интервалу вблизи частоты перехода $m-n$ и интервалу телесных углов, выделяемых приемником спонтанного излучения. Таким образом, для описания спонтанного испускания на переходе $m-n$ исходим из следующих кинетических уравнений для элементов матрицы плотности $[5,9]$ :

$$
\begin{gathered}
{\left[\frac{d}{d t}+A_{1}\right] \rho_{m}(\mathbf{v})=S_{m}(\mathbf{v})-2 \operatorname{Re}\left[i G_{1}^{*} \rho_{m n}(\mathbf{v})\right],} \\
{\left[\frac{d}{d t}+A_{2}\right] \rho_{k}(\mathbf{v})=S_{k}(\mathbf{v})-2 \operatorname{Re}\left[i G_{2}^{*} \rho_{k n}(\mathbf{v})\right],} \\
\frac{d}{d t} \rho_{n}(\mathbf{v})=S_{n}(\mathbf{v})+A_{1} \rho_{m}(\mathbf{v})+A_{2} \rho_{k}(\mathbf{v}) \\
+2 \operatorname{Re}\left[i\left(G_{1}^{*}+G_{\mu}^{*} e^{-i \varphi}\right) \rho_{m n}(\mathbf{v})\right]+2 \operatorname{Re}\left[i G_{2}^{*} \rho_{k n}(\mathbf{v})\right], \\
{\left[\frac{d}{d t}+\frac{A_{1}}{2}-i\left(\Omega_{01}-\mathbf{k}_{1} \mathbf{v}\right)\right] \rho_{m n}(\mathbf{v})=S_{m n}(\mathbf{v})} \\
+i G_{1}\left[\rho_{n}(\mathbf{v})-\rho_{m}(\mathbf{v})\right]-i G_{\mu} e^{i \varphi} \rho_{m}(\mathbf{v})-i G_{2} \rho_{m k}(\mathbf{v}), \\
{\left[\frac{d}{d t}+\frac{A_{2}}{2}-i\left(\Omega_{02}-\mathbf{k}_{2} \mathbf{v}\right)\right] \rho_{k n}(\mathbf{v})=S_{k n}(\mathbf{v})+i G_{2}\left[\rho_{n}(\mathbf{v})\right.} \\
\left.-\rho_{k}(\mathbf{v})\right]-i\left[G_{1}+G_{\mu} e^{i \varphi}\right] \rho_{k m}(\mathbf{v}),
\end{gathered}
$$




$$
\begin{aligned}
& \left\{\frac{d}{d t}+\frac{A_{1}+A_{2}}{2}-i\left[\Omega_{01}-\Omega_{02}-\left(\mathbf{k}_{1}-\mathbf{k}_{2}\right) \mathbf{v}\right]\right\} \\
& \times \rho_{m k}(\mathbf{v})=S_{m k}(\mathbf{v})+i G_{1} \rho_{n k}(\mathbf{v})-i G_{2}^{*} \rho_{m n}(\mathbf{v}),
\end{aligned}
$$

где

$$
\begin{gathered}
\varphi=\mathbf{q}_{1} \mathbf{r}-\varepsilon t, \quad \mathbf{q}_{1}=\mathbf{k}_{\mu}-\mathbf{k}_{1}, \quad \varepsilon=\omega_{\mu}-\omega_{1} \\
G_{1}=\frac{d_{m n} E_{1}}{2 \hbar}, \quad G_{2}=\frac{d_{k n} E_{2}}{2 \hbar}, \quad G_{\mu}=\frac{d_{m n} E_{\mu}}{2 \hbar} \\
\Omega_{01}=\omega_{1}-\omega_{m n}, \quad \Omega_{02}=\omega_{2}-\omega_{k n}, \quad \frac{d}{d t} \equiv \frac{\partial}{\partial t}+\mathbf{v} \nabla
\end{gathered}
$$

Здесь $\rho_{i}(\mathbf{v})$ - распределение частиц по скоростям на уровне $i(i=m, k, n) ; S_{i}(\mathbf{v})$ и $S_{i j}(\mathbf{v})$ - интегралы столкновений; $d_{m n}$ и $d_{k n}$ - матричные элементы дипольного момента переходов $m-n$ и $k-n$; $\omega_{\mu}$ - частота, на которой вычисляется спектральная плотность спонтанного испускания; $\mathbf{k}_{\mu}-$ волновой вектор спонтанного излучения.

Для интегралов столкновений в (11) будем использовать модель сильных столкновений $[5,9]$ :

$$
\begin{gathered}
S_{i}(\mathbf{v})=-v_{i} \rho_{i}(\mathbf{v})+v_{i} \rho_{i} W(\mathbf{v}), \quad i=m, k, n, \\
S_{m n}(\mathbf{v})=-v_{1} \rho_{m n}(\mathbf{v})+\widetilde{v}_{1} \rho_{m n} W(\mathbf{v}), \\
S_{k n}(\mathbf{v})=-v_{2} \rho_{k n}(\mathbf{v})+\widetilde{v}_{2} \rho_{k n} W(\mathbf{v}), \\
S_{m k}(\mathbf{v})=-v \rho_{m k}(\mathbf{v})+\widetilde{v} \rho_{m k} W(\mathbf{v}), \\
\rho_{j} \equiv \int \rho_{j}(\mathbf{v}) d \mathbf{v}, \quad j=m, k, n, m n, k n, m k,
\end{gathered}
$$

где $\mathrm{W}(\mathbf{v})$ - максвелловское распределение по скоростям; $v_{m}, v_{k}$ и $v_{n}$ - частоты упругих столкновений поглощающих частиц в возбужденных $(m, k)$ и основном (n) состояниях с буферными частицами; $v, v_{1}, v_{2}$ и $\widetilde{v}, \widetilde{v}_{1}, \widetilde{v}_{2}-$ „недиагональные“ частоты „ухода“ и „Прихода“ соответственно, являющиеся в общем случае комплексными величинами. Случаю, когда столкновения вызывают полную релаксацию когерентностей $\rho_{m k}(\mathbf{v})$, $\rho_{m n}(\mathbf{v}), \rho_{k n}(\mathbf{v})$ (отсутствие фазовой памяти при столкновениях на всех переходах), соответствует условие

$$
\widetilde{v}=\widetilde{v}_{1}=\widetilde{v}_{2}=0
$$

В случае отсутствия столкновительной релаксации когерентности $\rho_{k n}(\mathbf{v})$ (фазовая память при столкновениях сохраняется на переходе $k-n)$ частоты ухода $v_{2}$ и прихода $\widetilde{v}_{2}$ вещественны и равны между собой [5]:

$$
\widetilde{v}_{2}=v_{2}=v_{k}=v_{n} \equiv v_{\text {tr }},
$$

где $v_{\text {tr }}$ имеет смысл средней транспортной частоты упругих столкновений поглощающих частиц с буферными частицами [10]. Величина $v_{\text {tr }}$ связана соотношением $D=v_{T}^{2} / 2 v_{\operatorname{tr}}$ с коэффициентом диффузии $D$ поглощающих частиц в буферном газе, где $v_{T}-$ их наиболее вероятная скорость [11]. В случае отсутствия столкновительной релаксации когерентностей $\rho_{m n}(\mathbf{v})$ или $\rho_{m k}(\mathbf{v})$ (фазовая память при столкновениях сохраняется на переходах $m-n$ или $m-k$ ) выполняются соотношения $\widetilde{v}_{1}=v_{1}=v_{m}=v_{n}$ или $\widetilde{v}=v=v_{m}=v_{k}$ соответственно.

Далее ради упрощения задачи мы ограничимся рассмотрением случая слабого различия транспортных частот столкновений:

$$
\frac{\left|v_{m}-v_{n}\right|}{v_{n}} \ll 1, \quad \frac{\left|v_{k}-v_{n}\right|}{v_{n}} \ll 1
$$

При этом условии частоты столкновений $v_{m}, v_{k}, v_{n}$ можно заменить на среднюю транспортную частоту

$$
\bar{v}=\frac{v_{m}+v_{k}+v_{n}}{3} \approx \frac{v_{T}^{2}}{2 D} .
$$

Отметим, что в рамках условия (16) можно рассматривать столкновения любых типов: как сохраняющих фазу наведенного излучением дипольного момента, так и вызывающих сильный сбой фазы [7].

Решение уравнений (11) ищем в виде

$$
\begin{gathered}
\rho_{i}(\mathbf{v})=R_{i}(\mathbf{v})+2 \operatorname{R} e\left[r_{i}(\mathbf{v}) e^{i \varphi}\right], \quad i=m, k, n, \\
\rho_{i j}(\mathbf{v})=R_{i j}(\mathbf{v})+r_{i j}(\mathbf{v}) e^{i \varphi}+\widetilde{r}_{i j}(\mathbf{v}) e^{-i \varphi}, \\
i j=m n, m k, k n .
\end{gathered}
$$

Матричные элементы $R_{i}(\mathbf{v}), R_{i j}(\mathbf{v})$ отвечают решению задачи о взаимодействии с одними только сильными полями $E_{1}$ и $E_{2}$. Малые добавки $r_{i}(\mathbf{v}), r_{i j}(\mathbf{v}), \widetilde{r}_{i j}(\mathbf{v})$ обусловлены наличием слабого поля $E_{\mu}$.

В стационарных и пространственно однородных условиях система уравнений (11) при подстановке в нее (18) и с учетом (16) в первом приближении по $G_{\mu}$ разбивается на подсистему уравнений для $R_{i}(\mathbf{v}), R_{i j}(\mathbf{v})$ :

$$
\begin{gathered}
\left(A_{1}+\bar{v}\right) R_{m}(\mathbf{v})=\bar{v} R_{m} W(\mathbf{v})-2 \operatorname{Re}\left[i G_{1}^{*} R_{m n}(\mathbf{v})\right] \\
\left(A_{2}+\bar{v}\right) R_{k}(\mathbf{v})=\bar{v} R_{k} W(\mathbf{v})-2 \operatorname{Re}\left[i G_{2}^{*} R_{k n}(\mathbf{v})\right] \\
R_{m}(\mathbf{v})+R_{k}(\mathbf{v})+R_{n}(\mathbf{v})=\left(R_{m}+R_{k}+R_{n}\right) W(\mathbf{v}), \\
{\left[\gamma_{1}-i\left(\Omega_{01}-v_{1}^{\prime \prime}-\mathbf{k}_{1} \mathbf{v}\right)\right] R_{m n}(\mathbf{v})=\widetilde{v}_{1} R_{m n} W(\mathbf{v})} \\
+i G_{1}\left[R_{n}(\mathbf{v})-R_{m}(\mathbf{v})\right]-i G_{2} R_{m k}(\mathbf{v}) \\
{\left[\gamma_{2}-i\left(\Omega_{02}-v_{2}^{\prime \prime}-\mathbf{k}_{2} \mathbf{v}\right)\right] R_{k n}(\mathbf{v})=\widetilde{v}_{2} R_{k n} W(\mathbf{v})+} \\
+i G_{2}\left[R_{n}(\mathbf{v})-R_{k}(\mathbf{v})\right]-i G_{1} R_{k m}(\mathbf{v}) \\
{\left[\gamma-i\left(\Omega_{01}-\Omega_{02}-v^{\prime \prime}-\mathbf{q v}\right)\right] R_{m k}(\mathbf{v})} \\
=\widetilde{v} R_{m k} W(\mathbf{v})+i G_{1} R_{n k}(\mathbf{v})-i G_{2}^{*} R_{m n}(\mathbf{v})
\end{gathered}
$$

и подсистему уравнений для $r_{i}(\mathbf{v}), r_{i j}(\mathbf{v}), \widetilde{r}_{i j}(\mathbf{v})$ :

$$
\begin{aligned}
& {\left[A_{1}+\bar{v}-i\left(\varepsilon-\mathbf{q}_{1} \mathbf{v}\right)\right] r_{m}(\mathbf{v})=\bar{v} r_{m} W(\mathbf{v})} \\
& +i\left[G_{1} \widetilde{r}_{m n}^{*}(\mathbf{v})-G_{1}^{*} r_{m n}(\mathbf{v})\right], \\
& {\left[A_{2}+\bar{v}-i\left(\varepsilon-\mathbf{q}_{1} \mathbf{v}\right)\right] r_{k}(\mathbf{v})=\bar{v} r_{k} W(\mathbf{v})} \\
& +i\left[G_{2} \widetilde{r}_{k n}^{*}(\mathbf{v})-G_{2}^{*} r_{k n}(\mathbf{v})\right],
\end{aligned}
$$




$$
\begin{gathered}
{\left[\bar{v}-i\left(\varepsilon-\mathbf{q}_{1} \mathbf{v}\right)\right]\left[r_{m}(\mathbf{v})+r_{k}(\mathbf{v})+r_{n}(\mathbf{v})\right]} \\
=\bar{v}\left(r_{m}+r_{k}+r_{n}\right) W(\mathbf{v})-i G_{\mu} R_{m n}^{*}(\mathbf{v}), \\
{\left[\gamma_{1}-i\left(\Omega_{0 \mu}-\widetilde{v}_{1}^{\prime \prime}-\mathbf{k}_{\mu} \mathbf{v}\right)\right] r_{m n}(\mathbf{v})=\widetilde{v}_{1} r_{m n} W(\mathbf{v})} \\
+i G_{1}\left[r_{n}(\mathbf{v})-r_{m}(\mathbf{v})\right]-i G_{2} r_{m k}(\mathbf{v})-i G_{\mu} R_{m}(\mathbf{v}), \\
{\left[\gamma_{1}+i\left(\Omega_{01}-\widetilde{v}_{1}^{\prime \prime}-\varepsilon-\left(\mathbf{k}_{1}-\mathbf{q}_{1}\right) \mathbf{v}\right)\right] \widetilde{r}_{m n}^{*}(\mathbf{v})} \\
=\widetilde{v}_{1}^{*} \widetilde{r}_{m n}^{*} W(\mathbf{v})-i G_{1}^{*}\left[r_{n}(\mathbf{v})-r_{m}(\mathbf{v})\right]+i G_{2}^{*} \widetilde{r}_{m k}^{*}(\mathbf{v}), \\
{\left[\gamma_{2}-i\left(\Omega_{02}-\widetilde{v}_{2}^{\prime \prime}+\varepsilon-\left(\mathbf{k}_{2}+\mathbf{q}_{1}\right) \mathbf{v}\right)\right] r_{k n}(\mathbf{v})=\widetilde{v}_{2} r_{k n} W(\mathbf{v})} \\
+i G_{2}\left[r_{n}(\mathbf{v})-r_{k}(\mathbf{v})\right]-i G_{1} \widetilde{r}_{m k}^{*}(\mathbf{v})-i G_{\mu} R_{m k}^{*}(\mathbf{v}), \\
{\left[\gamma_{2}+i\left(\Omega_{02}-\widetilde{v}_{2}^{\prime \prime}-\varepsilon-\left(\mathbf{k}_{2}-\mathbf{q}_{1}\right) \mathbf{v}\right)\right] \widetilde{r}_{k n}^{*}(\mathbf{v})} \\
=\widetilde{v}_{2}^{*} \widetilde{r}_{k n}^{*} W(\mathbf{v})-i G_{2}^{*}\left[r_{n}(\mathbf{v})-r_{k}(\mathbf{v})\right]+i G_{1}^{*} r_{m k}(\mathbf{v}), \\
{\left[\gamma-i\left(\Omega_{01}-\Omega_{02}-v^{\prime \prime}+\varepsilon-\left(\mathbf{q}+\mathbf{q}_{1}\right) \mathbf{v}\right)\right] r_{m k}(\mathbf{v})} \\
=\widetilde{v}_{m k} W(\mathbf{v})+i G_{1} \widetilde{r}_{k n}^{*}(\mathbf{v})-i G_{2}^{*} r_{m n}(\mathbf{v}), \\
{\left[\gamma+i\left(\Omega_{01}-\Omega_{02}-v^{\prime \prime}-\varepsilon-\left(\mathbf{q}-\mathbf{q}_{1}\right) \mathbf{v}\right)\right] \widetilde{r}_{m k}^{*}(\mathbf{v})} \\
=\widetilde{v}^{*} \widetilde{r}_{m k}^{*} W(\mathbf{v})-i G_{1}^{*} r_{k n}(\mathbf{v})+i G_{2} \widetilde{r}_{m n}^{*}(\mathbf{v}) .
\end{gathered}
$$

Здесь введены следующие обозначения:

$$
\begin{gathered}
\gamma_{1}=\frac{A_{1}}{2}+v_{1}^{\prime}, \quad \gamma_{2}=\frac{A_{2}}{2}+v_{2}^{\prime}, \quad \gamma=\frac{A_{1}+A_{2}}{2}+v^{\prime}, \\
\Omega_{0 \mu}=\omega_{\mu}-\omega_{m n}, \quad \mathbf{q}=\mathbf{k}_{1}-\mathbf{k}_{2} .
\end{gathered}
$$

Верхние индексы „”“ и „”““ обозначают действительную и мнимую части комплексного числа. Величины $R_{i}$, $R_{i j}, r_{i}, r_{i j}, \widetilde{r}_{i j}^{*}$ в (19), (20) являются интегралами по скоростям от величин $R_{i}(\mathbf{v}), R_{i j}(\mathbf{v}), r_{i}(\mathbf{v}), r_{i j}(\mathbf{v}), \widetilde{r}_{i j}^{*}(\mathbf{v})$ соответственно $\left(R_{i} \equiv \int R_{i}(\mathbf{v}) d \mathbf{v}\right.$ и т.д.). В отсутствие фазовой памяти при столкновениях величины $v_{1}^{\prime} \equiv \operatorname{Re} v_{1}$ и $v_{2}^{\prime} \equiv \operatorname{Re} v_{2}$ определяют ударные полуширины линий поглощения на переходах $m-n$ и $k-n$ соответственно. Величины $\gamma_{1}$ и $\gamma_{2}$, являясь суммами радиационных и ударных полуширин, определяют однородные полуширины линий поглощения на переходах $m-n$ и $k-n$. В уравнениях (20) для $r_{i}(\mathbf{v}), r_{i j}(\mathbf{v}), \widetilde{r}_{i j}(\mathbf{v})$ величины $R_{i}(\mathbf{v})$, $R_{i j}(\mathbf{v})$ считаются заданными, так как они независимо определяются из замкнутых уравнений (19), которые описывают взаимодействие атома с одними только сильными полями.

Вероятность спонтанного испускания $w$ на частоте $\omega_{\mu}$ в расчете на один поглощающий атом, согласно общим правилам [5], определяется выражением

$$
w=\frac{2}{N} \operatorname{Re}\left[i G_{\mu}^{*} r_{m n}\right],
$$

где $N \equiv R_{n}+R_{m}+R_{k}-$ концентрация поглощающих частиц. Таким образом, в соответствии с поставленной задачей нам нужно найти величину $r_{m n}$ из системы уравнений (19), (20).

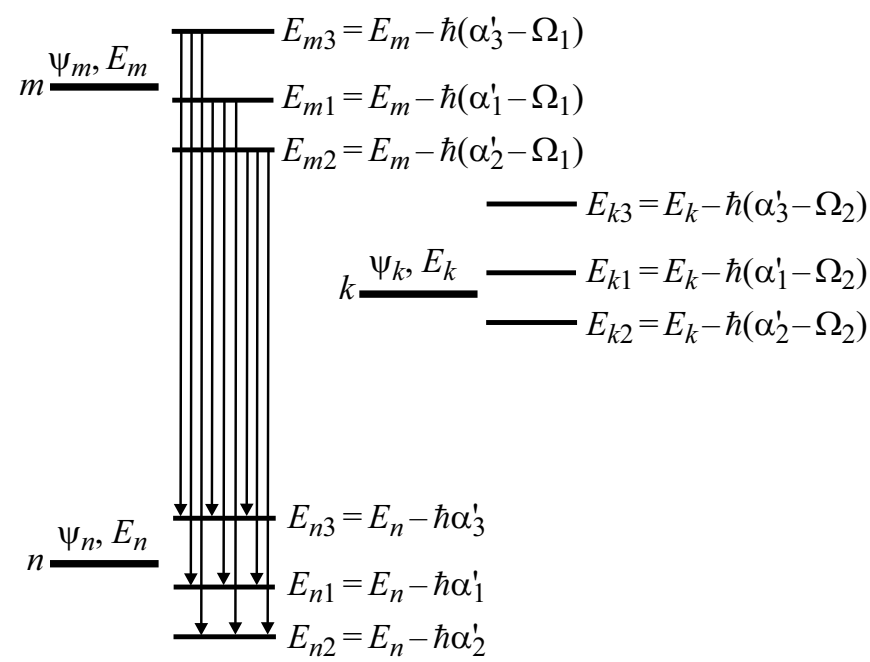

Рис. 2. К интерпретации спектра спонтанного испускания. Сплошными стрелками обозначены переходы между уровнями квазиэнергии.

\section{4. Слабоселективное по скоростям взаимодействие атомов с излучением}

Решение системы уравнений (19), (20) в общем случае произвольного соотношения между однородной и доплеровской ширинами линии поглощения приводит к громоздкому выражению для вероятности спонтанного испускания, которое поддается анализу только численными методами. Для упрощения задачи мы рассмотрим случай слабоселективного по скоростям взаимодействия атомов с излучением, когда распределение атомов по скоростям в состояниях $m, n, k$ мало отличается от максвелловского. Этот случай соответствует малости доплеровских ширин $k_{1} v_{T}, k_{2} v_{T}$ по сравнению с транспортной частотой столкновений:

$$
k_{1} v_{T}, k_{2} v_{T} \ll \bar{v} .
$$

Условие (23) позволяет применить очень простой и эффективный метод решения уравнений (18), (19) относительно интегральных по скоростям величин $R_{i}$, $R_{i j}, r_{i}, r_{i j}, \widetilde{r}_{i j}^{*}-$ метод предварительного усреднения по скоростям. Этот метод расчета, основанный на процедуре усреднения по скоростям в самих кинетических уравнениях для матрицы плотности, детально описан в работе [7]. Применяя данную процедуру усреднения по скоростям к уравнениям (19) и (20), аналогично тому, как это сделано в работе [7], получим:

$$
\begin{gathered}
A_{1} R_{m}=-2 \operatorname{Re}\left[i G_{1}^{*} R_{m n}\right], \\
A_{2} R_{k}=-2 \operatorname{Re}\left[i G_{2}^{*} R_{k n}\right], \\
\left(\Gamma_{1 a}-i \Omega_{1}\right) R_{m n}=i G_{1}\left(R_{n}-R_{m}\right)-i G_{2} R_{m k}, \\
\left(\Gamma_{2 a}+i \Omega_{2}\right) R_{n k}=-i G_{2}^{*}\left(R_{n}-R_{k}\right)+i G_{1}^{*} R_{m k},
\end{gathered}
$$



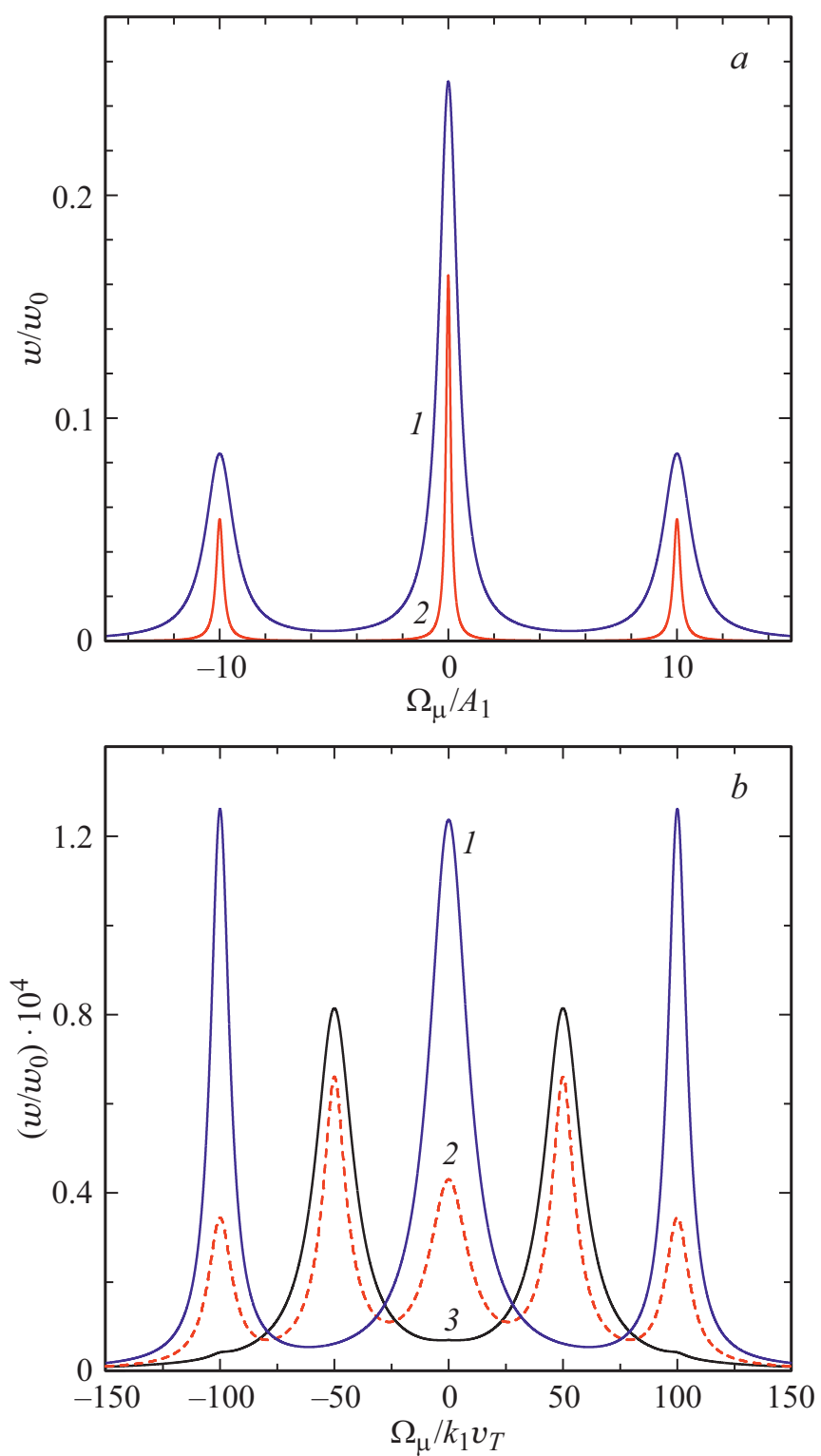

Рис. 3. Спектр спонтанного испускания на переходе $m-n$ при точном резонансе для возбуждающих полей $\left(\Omega_{1}=\Omega_{2}=0\right)$, $\left.A_{2}=0.1 A_{1}, a\right)$ - случай неподвижных и не испытывающих столкновений атомов $\left(v_{T}=0, \bar{v}=v_{1}=v_{2}=v=0\right)$, $G / A_{1}=5, \quad 1-\left|G_{1}\right| / A_{1}=5, \quad\left|G_{2}\right|=0,2-\left|G_{1}\right| / A_{1}=2$, $\left|G_{2}\right| / A_{1}=4.58$, линия рэлеевского рассеяния не учитывалась, $b)$ - случай столкновений, не сохраняющих фазовую память на всех переходах $\left(\widetilde{v}=\widetilde{v}_{1}=\widetilde{v}_{2}=0\right), \bar{v} / k_{1} v_{T}=10$, $v=v_{1}=v_{2}=\bar{v}, \quad A_{1} / k_{1} v_{T}=10^{-2}, \quad \theta_{1}=\pi / 2, \quad G / k_{1} v_{T}=50$, $1-\left|G_{1}\right| / k_{1} v_{T}=50, \quad\left|G_{2}\right|=0, \quad 2-\left|G_{1}\right| / k_{1} v_{T}=35$, $\left|G_{2}\right| / k_{1} v_{T}=35.71,3-\left|G_{1}\right| / k_{1} v_{T}=5, \quad\left|G_{2}\right| / k_{1} v_{T}=49.75$, линия рэлеевского рассеяния практически не заметна.

$$
\begin{gathered}
\left(\Gamma_{3 a}-i \varepsilon_{0}\right) R_{m k}=i G_{1} R_{n k}-i G_{2}^{*} R_{m n}, \\
\left(A_{1 a}-i \varepsilon\right) r_{m}=i G_{1} \widetilde{r}_{m n}^{*}-i G_{1}^{*} r_{m n}, \\
\left(A_{2 a}-i \varepsilon\right) r_{k}=i G_{2} \widetilde{r}_{k n}^{*}-i G_{2}^{*} r_{k n}, \\
\left(\Gamma_{q}-i \varepsilon\right)\left(r_{m}+r_{k}+r_{n}\right)=-i G_{\mu} R_{m n}^{*},
\end{gathered}
$$

$\left[\Gamma_{1 b}-i\left(\varepsilon+\Omega_{1}\right)\right] r_{m n}=i G_{1}\left(r_{n}-r_{m}\right)-i G_{2} r_{m k}-i G_{\mu} R_{m}$,

$$
\begin{gathered}
{\left[\Gamma_{1 c}-i\left(\varepsilon-\Omega_{1}\right)\right] \widetilde{r}_{m n}^{*}=-i G_{1}^{*}\left(r_{n}-r_{m}\right)+i G_{2}^{*} \widetilde{r}_{m k}^{*},} \\
{\left[\Gamma_{2 b}-i\left(\varepsilon+\Omega_{2}\right)\right] r_{k n}=i G_{2}\left(r_{n}-r_{k}\right)-i G_{1} \widetilde{r}_{m k}^{*}-i G_{\mu} R_{m k}^{*},} \\
{\left[\Gamma_{2 c}-i\left(\varepsilon-\Omega_{2}\right)\right] \widetilde{r}_{k n}^{*}=-i G_{2}^{*}\left(r_{n}-r_{k}\right)+i G_{1}^{*} r_{m k},} \\
{\left[\Gamma_{3 b}-i\left(\varepsilon+\varepsilon_{0}\right)\right] r_{m k}=i G_{1} \widetilde{r}_{k n}^{*}-i G_{2}^{*} r_{m n},} \\
{\left[\Gamma_{3 c}-i\left(\varepsilon-\varepsilon_{0}\right)\right] \widetilde{r}_{m k}^{*}=-i G_{1}^{*} r_{k n}+i G_{2} \widetilde{r}_{m n}^{*} .}
\end{gathered}
$$

Здесь введены следующие обозначения:

$$
\begin{gathered}
\Gamma_{1 a}=\Gamma_{1}+\frac{\left(k_{1} v_{T}\right)^{2} \gamma_{1}}{2\left[\gamma_{1}^{2}+\left(\Omega_{1}-\widetilde{v}_{1}^{\prime \prime}\right)^{2}\right]}, \\
\Gamma_{2 a}=\Gamma_{2}+\frac{\left(k_{2} v_{T}\right)^{2} \gamma_{2}}{2\left[\gamma_{2}^{2}+\left(\Omega_{2}-\widetilde{v}_{2}^{\prime \prime}\right)^{2}\right]}, \\
\Gamma_{3 a}=\Gamma_{3}+\frac{\left(q v_{T}\right)^{2} \gamma}{2\left[\gamma^{2}+\left(\varepsilon_{0}-\widetilde{v}^{\prime \prime}\right)^{2}\right]}, \quad A_{1 a}=A_{1}+\Gamma_{q}, \\
A_{2 a}=A_{2}+\Gamma_{q}, \quad \Gamma_{q}=\frac{\left(q_{1} v_{T}\right)^{2} \bar{v}}{2\left(\bar{v}^{2}+\varepsilon^{2}\right)}, \\
\Gamma_{1 b}=\Gamma_{1}+\frac{\left(k_{\mu} v_{T}\right)^{2} \gamma_{1}}{2\left[\gamma_{1}^{2}+\left(\varepsilon+\Omega_{1}-\widetilde{v}_{1}^{\prime \prime}\right)^{2}\right]}, \\
\Gamma_{1 c}=\Gamma_{1}+\frac{\left(\mathbf{k}_{1}-\mathbf{q}_{1}\right)^{2} v_{T}^{2} \gamma_{1}}{2\left[\gamma_{1}^{2}+\left(\varepsilon-\Omega_{1}+\widetilde{v}_{1}^{\prime \prime}\right)^{2}\right]}, \\
\Gamma_{2 b}=\Gamma_{2}+\frac{\left(\mathbf{k}_{2}+\mathbf{q}_{1}\right)^{2} v_{T}^{2} \gamma_{2}}{2\left[\gamma_{2}^{2}+\left(\varepsilon+\Omega_{2}-\widetilde{v}_{2}^{\prime \prime}\right)^{2}\right]}, \\
\Omega_{\mu}=\Omega_{0 \mu}-\left(v_{1}-\widetilde{v}_{1}\right)^{\prime \prime}, \quad \varepsilon_{0}=\Omega_{01}-\Omega_{02}-(v-\widetilde{v})^{\prime \prime} . \\
\Gamma_{2 c}=\Gamma_{2}+\frac{\left(\mathbf{k}_{2}-\mathbf{q}_{1}\right)^{2} v_{T}^{2} \gamma_{2}}{2\left[\gamma_{2}^{2}+\left(\varepsilon-\Omega_{2}+\widetilde{v}_{2}^{\prime \prime}\right)^{2}\right]}, \\
\Gamma_{3 b}=\Gamma_{3}+\frac{\left(\mathbf{q}+\mathbf{q}_{1}\right)^{2} v_{T}^{2} \gamma}{2\left[\gamma^{2}+\left(\varepsilon+\varepsilon_{0}-\widetilde{v}^{\prime \prime}\right)^{2}\right]}, \\
\Gamma_{3 c}=\Gamma_{3}+\frac{\left(\mathbf{q}-\mathbf{q}_{1}\right)^{2} v_{T}^{2} \gamma}{2\left[\gamma^{2}+\left(\varepsilon-\varepsilon_{0}+\widetilde{v}^{\prime \prime}\right)^{2}\right]}, \\
\left.2+(v-\widetilde{v})_{1}, \quad \widetilde{v}_{1}\right)^{\prime}, \quad \Gamma_{2}=\frac{A_{2}}{2}+\left(v_{2}-\widetilde{v}_{2}\right)^{\prime}, \\
A_{2}=\Omega_{02}-\left(v_{2}-\widetilde{v}_{2}\right)^{\prime \prime},
\end{gathered}
$$

Величины $\Gamma_{1}, \Gamma_{2}$ и $\Gamma_{3}$ в (25) зависят от степени coхранения фазовой памяти при столкновениях на переходах $m-n, k-n$ и $m-k$ соответственно. В отсутствие фазовой памяти на всех переходах (14) ввиду условия (23) выполняется соотношение $\Gamma_{1}, \Gamma_{2}, \Gamma_{3} \gg k_{1} v_{T}, k_{2} v_{T}$. В этом случае первые слагаемые $\Gamma_{i}$ в правых частях 


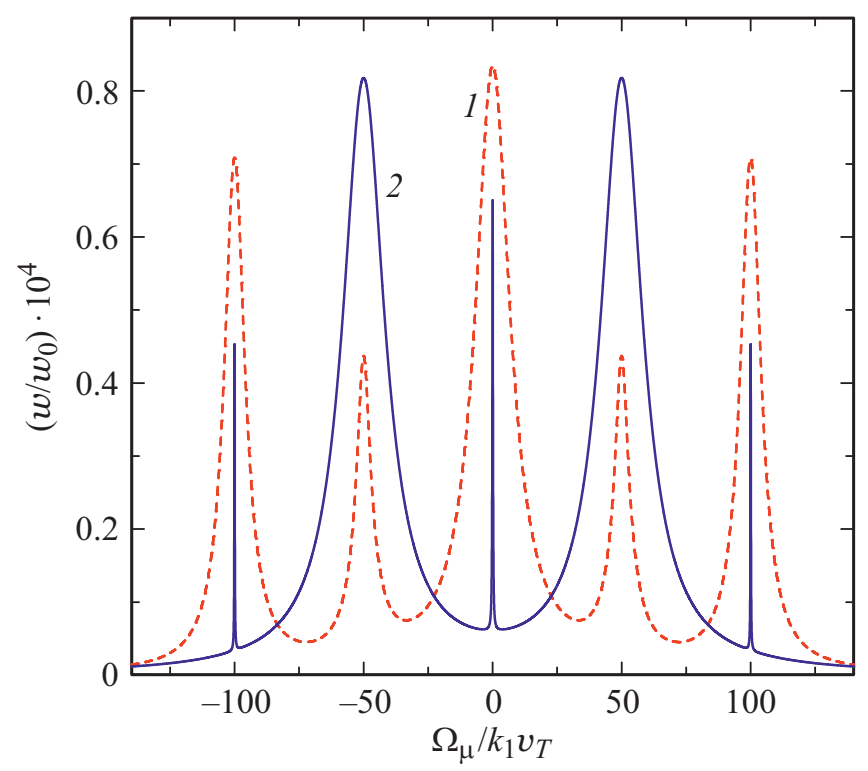

Рис. 4. Спектр спонтанного испускания на переходе $m-n$ в случае столкновений, сохраняющих фазовую память на смежном переходе $k-n\left(\widetilde{v}_{2}=v_{2}=\bar{v}\right)$ и не сохраняющих фазовую память на переходах $m-n$ и $m-k\left(\widetilde{v}_{1}=\widetilde{v}=0\right), \Omega_{1}=\Omega_{2}=0$, $A_{2}=0.1 A_{1}, \quad \bar{v} / k_{1} v_{T}=10, \quad v=v_{1}=v_{2}=\bar{v}, A_{1} / k_{1} v_{T}=10^{-2}$, $G / k_{1} v_{T}=50, k_{2} / k_{1}=0.7, \theta=0, \theta_{1}=\pi / 4,\left(\theta, \theta_{1}, \theta_{2}-\right.$ углы между волновыми векторами $\mathbf{k}_{1}$ и $\mathbf{k}_{2}, \mathbf{k}_{\mu}$ и $\mathbf{k}_{1}$ соответственно), $1-\left|G_{1}\right| / k_{1} v_{T}=45,\left|G_{2}\right| / k_{1} v_{T}=21.79,2-\left|G_{1}\right| / k_{1} v_{T}=5$, $\left|G_{2}\right| / k_{1} v_{T}=49.75$.

формул для $\Gamma_{i a}, \Gamma_{i b}$ и $\Gamma_{i c}(i=1,2,3)$ становятся главными, и поэтому в этих формулах вторыми слагаемыми, пропорциональными $v_{T}^{2}$, можно пренебречь. При сохранении фазовой памяти на всех переходах выполняется соотношение $\Gamma_{1}, \Gamma_{2}, \Gamma_{3} \ll k_{1} v_{T}, k_{2} v_{T}$, и в этом случае главными в формулах для $\Gamma_{i a}, \Gamma_{i b}$ и $\Gamma_{i c}(i=1,2,3)$ будут последние слагаемые.

\section{5. Анализ спектра резонансной флуоресценции}

Решая систему уравнений (24) стандартным путем, мы получили аналитическое выражение для расчета вероятности спонтанного испускания $w(22)$ на частоте $\omega_{\mu}$. Конечное выражение для $w$ мы здесь не приводим ввиду его громоздкости.

При достаточно высоких интенсивностях возбуждающих излучений и точного резонанса для сильных полей,

$$
\begin{gathered}
G \equiv \sqrt{\left|G_{1}\right|^{2}+\left|G_{2}\right|^{2}} \gg \Gamma_{i a}, \Gamma_{i b}, \Gamma_{i c}, A_{1 a}, A_{2 a}, \\
i=1,2,3, \quad \Omega_{1}=\Omega_{2}=0
\end{gathered}
$$

из точного выражения для $w$ следует, что спектр резонансной флуоресценции описывается формулой

$$
\begin{aligned}
& w=\left|G_{\mu}\right|^{2} \frac{\left|G_{1}\right|^{2}}{G^{2}} \frac{\Delta_{2}}{\Delta_{1}}\left\{\frac{\Delta_{3} E_{0}}{4 \Delta_{2} E_{2}} \frac{A_{1}}{\Gamma_{e f}} \frac{\gamma_{q}}{\gamma_{q}^{2}+\varepsilon^{2}}+\frac{\Gamma_{e f}}{\Gamma_{e f}^{2}+\varepsilon^{2}}\right. \\
& +\frac{\Gamma_{e f}^{(1)} / 2}{\left(\Gamma_{e f}^{(1)}\right)^{2}+(\varepsilon+2 G)^{2}}+\frac{\Gamma_{e f}^{(1)} / 2}{\left(\Gamma_{e f}^{(1)}\right)^{2}+(\varepsilon-2 G)^{2}} \\
& +\frac{2\left|G_{2}\right|^{4}\left[v^{\prime}-\widetilde{v}^{\prime}+\left(q v_{T}\right)^{2} /(2 \gamma)\right]}{\Delta_{2}} \\
& \times\left[\frac{K \Gamma_{\beta}+\Gamma_{f}(\varepsilon+G)^{2}}{\left[K-(\varepsilon+G)^{2}\right]^{2}+\left(\Gamma_{\beta}+\Gamma_{f}\right)^{2}(\varepsilon+G)^{2}}\right. \\
& \left.\left.+\frac{K \Gamma_{\beta}+\Gamma_{f}(\varepsilon-G)^{2}}{\left[K-(\varepsilon-G)^{2}\right]^{2}+\left(\Gamma_{\beta}+\Gamma_{f}\right)^{2}(\varepsilon-G)^{2}}\right]\right\} .
\end{aligned}
$$

Здесь введены следующие обозначения:

$$
\begin{aligned}
& \Gamma_{\mathrm{ef}}=\frac{\left|G_{1}\right|^{2} \widetilde{\Gamma}_{1}+\left|G_{2}\right|^{2} \widetilde{\Gamma}_{2}}{G^{2}}, \\
& \Gamma_{\mathrm{ef}}^{(1)}=\frac{\left|G_{1}\right|^{2} \widetilde{\Gamma}_{3}+\left|G_{2}\right|^{2} \widetilde{\Gamma}_{4}}{G^{2}}+\frac{\left|G_{1}\right|^{2}\left|G_{2}\right|^{2} \widetilde{\Gamma}_{5}}{G^{4}}, \\
& \widetilde{\Gamma}_{1}=\Gamma_{1}+\frac{\left(3 k_{1}^{2}-2 \mathbf{k}_{1} \mathbf{k}_{\mu}\right) v_{T}^{2} \gamma_{1}}{2\left[\gamma_{1}^{2}+\left(\widetilde{v}_{1}^{\prime \prime}\right)^{2}\right]} \\
& \widetilde{\Gamma}_{2}=\Gamma_{2}+\frac{\left(2 k_{1}^{2}+k_{2}^{2}-2 \mathbf{k}_{1} \mathbf{k}_{\mu}\right) v_{T}^{2} \gamma_{2}}{2\left[\gamma_{2}^{2}+\left(\widetilde{v}_{2}^{\prime \prime}\right)^{2}\right]}, \\
& \widetilde{\Gamma}_{3}=\frac{\Gamma_{1}+A_{1}}{2}+\frac{v_{T}^{2}}{4}\left[\frac{q_{1}^{2} \bar{v}}{\bar{v}^{2}+4 G^{2}}+\frac{\left(3 k_{1}^{2}-2 \mathbf{k}_{1} \mathbf{k}_{\mu}\right) \gamma_{1}}{\gamma_{1}^{2}+4 G^{2}}\right], \\
& \widetilde{\Gamma}_{4}=\frac{\Gamma_{2}+A_{2}}{2}+\frac{v_{T}^{2}}{4}\left[\frac{q_{1}^{2} \bar{v}}{\bar{v}^{2}+4 G^{2}}+\frac{\left(2 k_{1}^{2}+k_{2}^{2}-2 \mathbf{k}_{1} \mathbf{k}_{\mu}\right) \gamma_{2}}{\gamma_{2}^{2}+4 G^{2}}\right] \text {, } \\
& \widetilde{\Gamma}_{5}=\frac{v^{\prime}-\widetilde{v}^{\prime}}{2}+\frac{v_{T}^{2}}{4}\left[\frac{\left(q_{1}^{2}+q^{2}\right) \gamma}{\gamma^{2}+4 G^{2}}-\frac{q_{1}^{2} \bar{v}}{\bar{v}^{2}+4 G^{2}}\right] \\
& \gamma_{q}=\frac{\left(q_{1} v_{T}\right)^{2}}{2 \bar{v}} \\
& \Gamma_{\beta}=\left(G^{-4} / 2\right)\left\{\left[\left|G_{1}\right|^{4}\left(\Gamma_{2 c}+\Gamma_{3 b}\right)+\left|G_{2}\right|^{4}\left(\Gamma_{1 c}+\Gamma_{3 c}\right)\right.\right. \\
& \left.\left.+\left|G_{1}\right|^{2}\left|G_{2}\right|^{2}\left(A_{1 a}+A_{2 a}+\Gamma_{1 c}+\Gamma_{2 c}\right)\right]\right\}, \\
& \Gamma_{f}=\left(G^{-4} / 2\right)\left\{\left[\left|G_{1}\right|^{4}\left(\Gamma_{2 b}+\Gamma_{3 c}\right)+\left|G_{2}\right|^{4}\left(\Gamma_{1 b}+\Gamma_{3 b}\right)\right.\right. \\
& \left.\left.+\left|G_{1}\right|^{2}\left|G_{2}\right|^{2}\left(A_{1 a}+A_{2 a}+\Gamma_{1 b}+\Gamma_{2 b}\right)\right]\right\},
\end{aligned}
$$




$$
\begin{gathered}
K=\left(G^{-6} / 4\right)\left\{\left|G_{1}\right|^{6}\left(\Gamma_{2 c}+\Gamma_{3 b}\right)\left(\Gamma_{2 b}+\Gamma_{3 c}\right)+\left|G_{2}\right|^{6}\right. \\
\times\left(\Gamma_{1 b}+\Gamma_{3 b}\right)\left(\Gamma_{1 c}+\Gamma_{3 c}\right)+\left|G_{1}\right|^{2}\left|G_{2}\right|^{4} \\
\times\left[\left(A_{1 a}+A_{2 a}\right)\left(\Gamma_{1 b}+\Gamma_{1 c}+\Gamma_{3 b}+\Gamma_{3 c}\right)+\Gamma_{1 b}\left(\Gamma_{1 c}+\Gamma_{2 c}\right)\right. \\
\left.+\Gamma_{2 b}\left(\Gamma_{1 c}+\Gamma_{3 c}\right)+\Gamma_{3 b}\left(\Gamma_{2 c}-\Gamma_{3 c}\right)\right]+\left|G_{1}\right|^{4}\left|G_{2}\right|^{2} \\
\times\left[\left(A_{1 a}+A_{2 a}\right)\left(\Gamma_{2 b}+\Gamma_{2 c}+\Gamma_{3 b}+\Gamma_{3 c}\right)\right. \\
\left.\left.+\Gamma_{1 b}\left(\Gamma_{2 c}+\Gamma_{3 b}\right)+\Gamma_{2 b}\left(\Gamma_{1 c}+\Gamma_{2 c}\right)+\Gamma_{3 c}\left(\Gamma_{1 c}-\Gamma_{3 b}\right)\right]\right\}, \\
\Delta_{1}=2\left|G_{1}\right|^{4} A_{2}+2\left|G_{2}\right|^{4} A_{1}+\left|G_{1}\right|^{2}\left|G_{2}\right|^{2}\left(6 \Gamma_{3 a}-A_{1}-A_{2}\right), \\
\Delta_{2}=\left|G_{1}\right|^{4} A_{2}+\left|G_{2}\right|^{4} A_{1}+2\left|G_{1}\right|^{2}\left|G_{2}\right|^{2} \Gamma_{3 a}, \\
\Delta_{3}=\left|G_{1}\right|^{2} A_{2}+\left|G_{2}\right|^{2}\left(2 \Gamma_{3 a}-A_{2}\right), \\
E_{0}=\left|G_{1}\right|^{4} A_{1 a} A_{2 a} \Gamma_{1 c}+\left|G_{2}\right|^{4} A_{1 a}\left[\Gamma_{2 c} \Gamma_{3 b}+\Gamma_{2 b}\left(\Gamma_{3 b}-A_{2 a}\right)\right] \\
+\left|G_{1}\right|^{2}\left|G_{2}\right|^{2}\left\{A_{2 a}\left[\Gamma_{2 c} \Gamma_{3 b}-\Gamma_{2 b}\left(\Gamma_{3 c}-A_{1 a}\right)\right]\right. \\
\left.+A_{1 a} \Gamma_{1 c}\left(\Gamma_{3 b}+\Gamma_{3 c}-A_{2 a}\right)\right\}, \\
E_{2}=\left|G_{1}\right|^{4} A_{2 a}+\left|G_{2}\right|^{4} A_{1 a}+\left|G_{1}\right|^{2}\left|G_{2}\right|^{2} \\
\times\left(3 \Gamma_{3 b}+3 \Gamma_{3 c}-A_{1 a}-A_{2 a}\right) / 2 .
\end{gathered}
$$

Выражение (27) состоит из суммы шести вкладов. Первый член в фигурных скобках, пропорциональный $\gamma_{q}$, описывает узкую спектральную компоненту лоренцевой формы (линия рэлеевского рассеяния) с полушириной $\gamma_{q}$, центрированную на частоте $\omega_{1}$ возбуждающего поля $\left|G_{1}\right|$. В частном случае $\gamma_{q} \rightarrow 0$ (это случай $\mathbf{k}_{\mu}=\mathbf{k}_{1}$ для движущихся атомов, либо случай неподвижных атомов, $\left.v_{T}=0\right)$ этот член определяется $\delta$-функцией и описывает упругое рассеяние света атомами. Хотя этот член стремится к бесконечности при $\varepsilon=0, \gamma_{q} \rightarrow 0$, его вклад в энергию спонтанного испускания (результат интегрирования по $\varepsilon$ ) в условиях (26) пренебрежимо мал. В спектре резонансной флуоресценции при условии высоких интенсивностей возбуждающих излучений линия рэлеевского рассеяния становится заметной лишь при малых углах $\theta_{1}$ между волновыми векторами $\mathbf{k}_{\mu}$ и $\mathbf{k}_{1}$. Как полуширина $\gamma_{q}$, так и амплитуда линии рэлеевского рассеяния анизотропны по отношению к взаимной ориентации волновых векторов спонтанного и возбуждающего излучений. В условиях, когда линия рэлеевского рассеяния регистрируется надежно, по ее полуширине $\gamma_{q}$ можно определять коэффициент диффузии $D$ или транспортную частоту столкновений $\bar{v}$ поглощающих частиц с буферными:

$$
D=\frac{\gamma_{q}}{\left(\mathbf{k}_{\mu}-\mathbf{k}_{1}\right)^{2}}, \quad \bar{v}=\frac{v_{T}^{2}}{2 D} .
$$

Второй, третий и четвертый члены в фигурных скобках выражения (27) описывают спектральные компоненты лоренцевой формы с максимумами на частотах $\omega_{\mu}=\omega_{1}$ и $\omega_{\mu}=\omega_{1} \pm 2 G$ и полуширинами соответственно $\Gamma_{\mathrm{ef}}$ и $\Gamma_{\mathrm{ef}}^{(1)}$. Энергия (интегральная интенсивность)
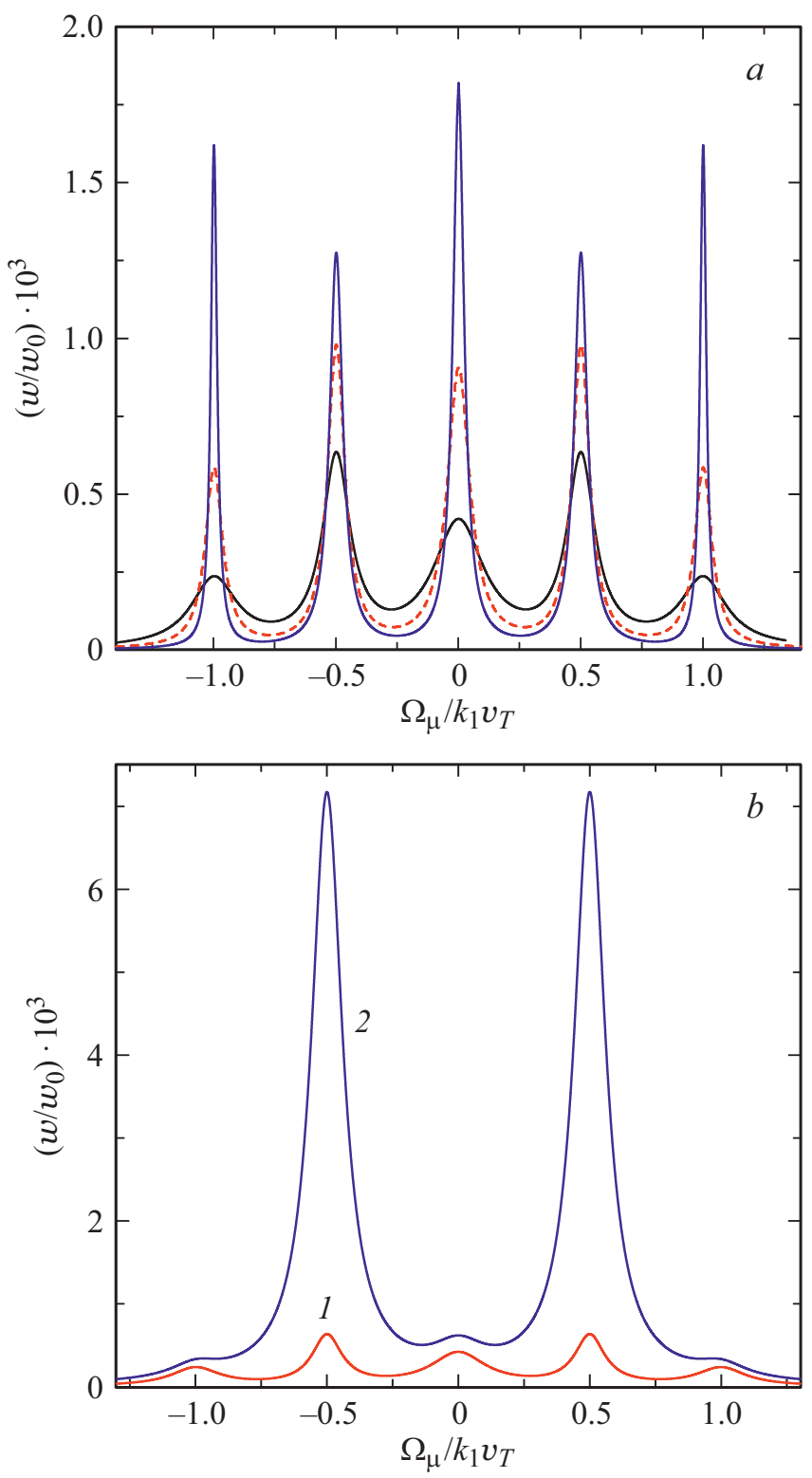

Рис. 5. Спектр спонтанного испускания на переходе $m-n$ в случае столкновений, сохраняющих фазовую память на всех переходах $\quad\left(v=\widetilde{v}=v_{1}=\widetilde{v}_{1}=v_{2}=\widetilde{v}_{2}=\bar{v}\right), \quad \Omega_{1}=\Omega_{2}=0$, $A_{2}=0.1 A_{1}, \quad A_{1} / k_{1} v_{T}=10^{-2}, \quad \bar{v} / k_{1} v_{T}=10, \quad k_{2} / k_{1}=0.7$, $G / k_{1} v_{T}=0.5, \quad\left|G_{1}\right| / k_{1} v_{T}=0.1, \quad\left|G_{2}\right| / k_{1} v_{T}=0.49 ; \quad$ a) $\theta=0, \theta_{1}=\pi / 18, \pi / 4, \pi / 2$ (значения угла $\theta_{1}$ между $\mathbf{k}_{\mu}$ и $\mathbf{k}_{1}$ соответствуют расположению кривых в порядке сверху вниз в окрестности $\left.\left.\Omega_{\mu}=0\right) ; b\right)-\theta_{1}=\theta_{2}=\pi / 2,1-\theta=0,2-$ $\theta=\pi\left(\theta-\right.$ угол между $\mathbf{k}_{1}$ и $\left.\mathbf{k}_{2}\right)$.

центральной компоненты на частоте $\omega_{\mu}=\omega_{1}$ в два раза больше энергии каждой из боковых компонент на частотах $\omega_{\mu}=\omega_{1} \pm 2 G$. Отношение $A_{2 G}$ амплитуды каждой из боковых компонент к амплитуде центральной компоненты (без учета узкой компоненты с полушириной $\gamma_{q}$ ), как видно из (27), равно

$$
A_{2 G}=\frac{\Gamma_{\mathrm{ef}}}{2 \Gamma_{\mathrm{ef}}^{(1)}}
$$


Как полуширины, так и амплитуды спектральных компонент зависят от интенсивностей возбуждающих излучений, частоты столкновений, степени сохранения фазовой памяти при столкновениях на переходах $m-n, k-n$ и $m-k$, взаимной ориентации волновых векторов $\mathbf{k}_{1}, \mathbf{k}_{2}$ и $\mathbf{k}_{\mu}$.

Пятый и шестой члены в фигурных скобках выражения (27) описывают внутренние боковые спектральные компоненты сложной (не лоренцевой) формы с максимумами на частотах $\omega_{\mu}=\omega_{1} \pm G$. Их полуширина на полувысоте $\Gamma_{G}$ дается выражением

$$
\begin{gathered}
\Gamma_{G}=\left(\sqrt{B^{2}+K^{2}}-B\right)^{1 / 2}, \\
B=\frac{\Gamma_{\beta}+\Gamma_{f}}{2 \Gamma_{\beta}}\left[\left(\Gamma_{\beta}+\Gamma_{f}\right) \Gamma_{\beta}-2 K\right] .
\end{gathered}
$$

Отношение $E_{G}$ энергии каждой из внутренних боковых компонент к энергии центральной компоненты, как следует из формулы (27), равно

$$
E_{G}=2\left|G_{2}\right|^{4}\left[v^{\prime}-\widetilde{v}^{\prime}+\left(q v_{T}\right)^{2} /(2 \gamma)\right] / \Delta_{2} .
$$

Отношение $A_{G}$ амплитуды каждой из внутренних боковых компонент к амплитуде центральной компоненты равно

$$
A_{G}=E_{G} \Gamma_{\beta} \Gamma_{e f} / K
$$

Внутренние боковые спектральные компоненты возникают только при наличии второго возбуждающего поля (при $\left|G_{2}\right| \neq 0$ ) благодаря столкновительной релаксации когерентности $\rho_{m k}$ (слагаемое $v^{\prime}-\widetilde{v}^{\prime}$ в факторе $v^{\prime}-\widetilde{v}^{\prime}+\left(q v_{T}\right)^{2} /(2 \gamma)$, которому пропорциональна амплитуда компоненты) и движению атомов (слагаемое $\left.\left(q v_{T}\right)^{2} /(2 \gamma)\right)$. Без учета движения и столкновений атомов эти компоненты не возникают. Амплитуда компонент максимальна в случае отсутствия фазовой памяти при столкновениях на переходе $m-k\left(\right.$ при $\left.\widetilde{v}^{\prime}=0\right)$ и минимальна в случае полного сохранения фазовой памяти при столкновениях на переходе $m-k$ (при $\left.\widetilde{v}^{\prime}=v^{\prime}\right)$.

Согласно (27), при высоких интенсивностях возбуждающих излучений и точном резонансе для сильных полей в спектре флуоресценции трехуровневых атомов присутствуют пять спектральных компонент. При отсутствии второго возбуждающего поля (при $\left|G_{2}\right|=0$ ) формула (27) описывает триплет резонансной флуоресценции двухуровневых атомов с учетом столкновений и теплового движения атомов, как это сделано в [12].

Обратим внимание на следующее обстоятельство. Из очевидных физических представлений следует, что полная (по частоте и по углам) интенсивность спонтанного испускания на заданном переходе определяется заселенностью верхнего уровня и первым коэффициентом Эйнштейна для этого перехода. Следовательно, интеграл по частоте от спектральной плотности $w$ спонтанного испускания должен сводиться (с точностью до константы) к заселенности уровня $m$. Это требование полезно с точки зрения проверки правильности вычислений. В

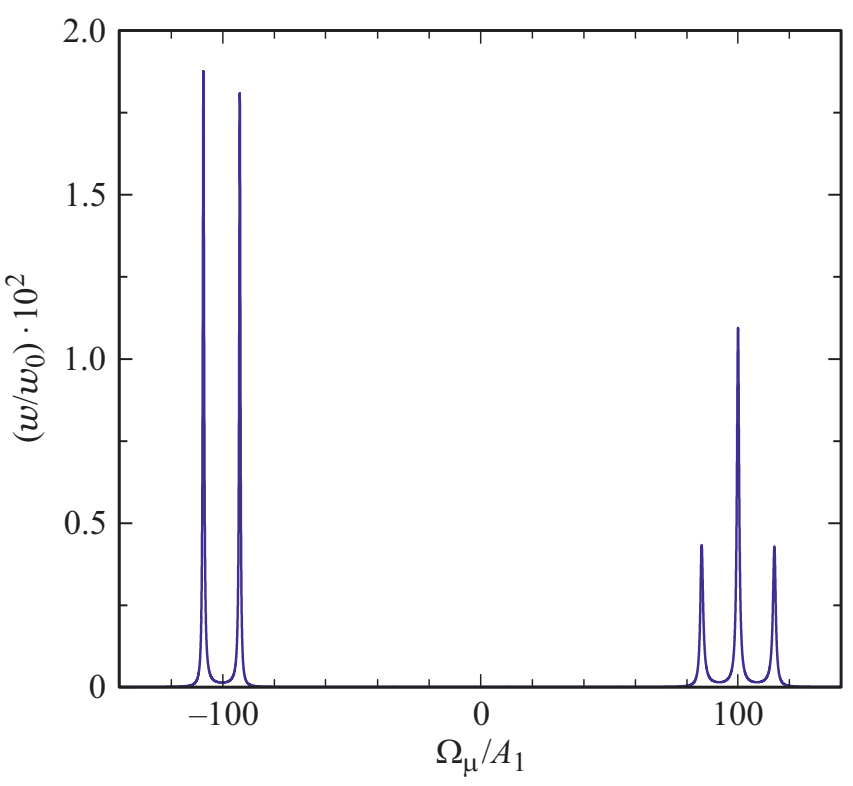

Рис. 6. Спектр спонтанного испускания неподвижных и не испытывающих столкновений атомов $\left(v_{T}=0\right.$, $\left.\bar{v}=v_{1}=v_{2}=v=0\right) \quad$ при высокой интенсивности возбуждающего излучения на переходе $k-n$ и при оптимальной отстройке частоты возбуждающего излучения на переходе $m-n ; \quad A_{2}=0.1 A_{1},\left|G_{1}\right| / A_{1}=10,\left|G_{2}\right| / A_{1}=100$, $\Omega_{1}=\left|G_{2}\right|, \Omega_{2}=0$.

частности, в условиях применимости результата (27) заселенность уровня $m$ дается выражением

$$
R_{m}=N\left|G_{1}\right|^{2} \Delta_{3} / \Delta_{1},
$$

которое следует из решения системы первых пяти уравнений (24) при высоких интенсивностях полей возбуждающих излучений и $\Omega_{1}=\Omega_{2}=0$. Интегрирование в $(27)$ по $\varepsilon$ приводит к соотношению

$$
\int_{-\infty}^{\infty} w d \varepsilon=2 \pi\left|G_{\mu}\right|^{2} \frac{R_{m}}{N} .
$$

Мы убедились также, что данное соотношение выполняется и в общем случае решения уравнений (24), как и должно быть.

Приведем несколько характерных примеров спектров резонансной флуоресценции, описываемых формулой (27). Они представлены на рис. 3-5 для разных значений интенсивностей возбуждающих излучений, частоты столкновений и степени сохранения фазовой памяти при столкновениях на переходах $m-n, k-n$ и $m-k$. Для нормировки спектров на всех рисунках используется величина $w_{0}$ :

$$
w_{0}=\frac{4\left|G_{\mu}\right|^{2}}{A_{1}},
$$

которая есть вероятность спонтанного испускания в центре линии на переходе $m-n$ неподвижным и не 
испытывающим столкновений атомом, находящимся на возбужденном уровне $m$, в отсутствие возбуждающих излучений.

Когда движение и столкновения атомов не учитываются $\left(v_{T}=0, \bar{v}=v_{1}=v_{2}=v=0\right)$, то из формул (27), (28) получаем выражение для спектра резонансной флуоресценции, полученное в [3] (правда, без учета $\delta$ функции рэлеевского рассеяния):

$$
\begin{aligned}
& w=\left|G_{\mu}\right|^{2} \frac{\left|G_{1}\right|^{2}}{2 G^{2}}\left\{C \delta(\varepsilon)+\frac{\Gamma_{\mathrm{ef}}}{\Gamma_{\mathrm{ef}}^{2}+\varepsilon^{2}}\right. \\
& \left.+\frac{3 \Gamma_{\mathrm{ef}} / 4}{\left(3 \Gamma_{\mathrm{ef}} / 2\right)^{2}+(\varepsilon+2 G)^{2}}+\frac{3 \Gamma_{\mathrm{ef}} / 4}{\left(3 \Gamma_{\mathrm{ef}} / 2\right)^{2}+(\varepsilon-2 G)^{2}}\right\},
\end{aligned}
$$

где

$$
\Gamma_{\mathrm{ef}}=\frac{\left|G_{1}\right|^{2} A_{1}+\left|G_{2}\right|^{2} A_{2}}{2 G^{2}},
$$

множитель $C$ в члене, ответственном за рэлеевское рассеяние, зависит от $\left|G_{1}\right|,\left|G_{2}\right|, A_{1}, A_{2}$. Выражение для него довольно громоздко и мы его не приводим, учитывая также очень малый энергетический вклад рэлеевской линии в общий спектр. В остальном спектр резонансной флуоресценции, согласно (37), состоит из трех линий (рис. 3,a). Центральная линия имеет полуширину $\Gamma_{\mathrm{ef}}$, а боковые линии в полтора раза шире. Амплитуды боковых линий в три раза меньше амплитуды центральной линии: $A_{2 G}=1 / 3$. Полуширины линий зависят от атомных параметров и от относительной интенсивности полей. При $\left|G_{2}\right| \ll\left|G_{1}\right|$ (слабая интенсивность возбуждающего излучения на смежном переходе $k-n)$ полуширина центральной линии $\Gamma_{\text {ef }}=A_{1} / 2$. При $\left|G_{2}\right| \gg\left|G_{1}\right|$ (высокая интенсивность возбуждающего излучения на смежном переходе) полуширина $\Gamma_{\mathrm{ef}}=A_{2} / 2$. В случае $A_{2} \ll A_{1}$ все линии спонтанного испускания многократно сужаются с ростом интенсивности возбуждающего излучения на смежном переходе (ср. кривые 1 и 2 на рис. $3, a)$.

Как следует из более общего результата (27), столкновения радикально изменяют спектр спонтанного испускания. На рис. $3, b$ показаны спектры в случае столкновений, не сохраняющих фазовую память на всех переходах $\left(\widetilde{v}=\widetilde{v}_{1}=\widetilde{v}_{2}=0\right)$. При $\left|G_{2}\right|=0$ (атом возбуждается единственным полем, резонансным переходу $m-n)$ в спектре присутствуют три компоненты (так называемый триплет Моллоу [8]): центральная компонента на частоте $\omega_{\mu}=\omega_{1}$ и две боковые компоненты на частотах $\omega_{\mu}=\omega_{1} \pm 2|G|$ (кривая 1 на рис. $\left.3, b\right)$. При возбуждении атома двумя полями в спектре спонтанного испускания возникают еще две внутренние боковые спектральные компоненты на частотах $\omega_{\mu}=\omega_{1} \pm G$ (кривая 2 на рис. $3, b)$. При $\left|G_{2}\right| \gg\left|G_{1}\right|$ в спектре остаются только две внутренние боковые спектральные компоненты, амплитуды центральной и внешних боковых компонент малы и эти компоненты практически не заметны (кривая 3 на рис. $3, b)$. Полуширины всех линий спектра резонансной флуоресценции слабо зависят от относительной интенсивности полей излучения и примерно равны ударным полуширинам линий поглощения на переходах $m-n$ и $k-n$. Анизотропия спектра по отношению к взаимной ориентации волновых векторов $\mathbf{k}_{1}, \mathbf{k}_{2}$ и $\mathbf{k}_{\mu}$ практически не заметна.

На рис. 4 показан спектр спонтанного испускания на переходе $m-n$ в случае столкновений, сохраняющих фазовую память на смежном переходе $k-n\left(\widetilde{v}_{2}=v_{2}=\bar{v}\right.$, отсутствие столкновительной релаксации когерентности $\left.\rho_{k n}\right)$ и не сохраняющих фазовую память на переходах $m-n$ и $m-k\left(\widetilde{v}_{1}=\widetilde{v}=0\right)$. Полуширины линий спектра сильно зависят от относительной интенсивности возбуждающих полей. При $\left|G_{2}\right|<\left|G_{1}\right|$ полуширины всех линий спектра по порядку величины сопоставимы с ударной полушириной линии поглощения на переходе $m-n$, анизотропия спектра испускания по отношению к взаимной ориентации волновых векторов $\mathbf{k}_{1}, \mathbf{k}_{2}$ и $\mathbf{k}_{\mu}$ практически не заметна (кривая 1 на рис. 4). С ростом интенсивности излучения, резонансного смежному переходу $k-n$ (при $\left.\left|G_{2}\right| \gg\left|G_{1}\right|\right)$, происходит резкое сужение центральной и внешних боковых линий (кривая 2 на рис. 4). В случае почти полного сохранения фазовой памяти в столкновениях на переходе $k-n$ (при $\left.\left(v_{2}-\widetilde{v}_{2}\right)^{\prime} \ll A_{2} / 2\right)$ и при $\left|G_{2}\right| \gg\left|G_{1}\right|$ для полуширин центральной и боковых линий в (27) имеем (при $\left.\bar{v} \gg A_{1}, A_{2}\right)$ :

$$
\begin{gathered}
\Gamma_{\mathrm{ef}}=\frac{A_{2}}{2}+\left(2 k_{1}^{2}+k_{2}^{2}-2 \mathbf{k}_{1} \mathbf{k}_{\mu}\right) D, \\
\Gamma_{\mathrm{ef}}^{(1)}=\frac{3}{4} A_{2}+\left(4 k_{1}^{2}+k_{2}^{2}-4 \mathbf{k}_{1} \mathbf{k}_{\mu}\right) D \frac{\bar{v}^{2}}{8\left|G_{2}\right|^{2}}, \\
\Gamma_{G}=\frac{\gamma_{1}+\gamma}{2} \gg \Gamma_{\mathrm{ef}}, \Gamma_{\mathrm{ef}}^{(1)} .
\end{gathered}
$$

Согласно формуле (39), полуширины центральной и внешних боковых линий (и, следовательно, амплитуды линий, которые обратно пропорциональны их полуширинам) в спектре резонансной флуоресценции зависят от коэффициента диффузии $D$ частиц и анизотропны по отношению к взаимной ориентации волновых векторов спонтанного и резонансного переходу $m-n$ возбуждающего излучения. Анизотропия особенно ярко проявляется для центральной линии при $A_{2} \ll k_{2}^{2} D$, когда полуширина линии прямо пропорциональна коэффициенту диффузии. В этом случае при однонаправленных $\left(\mathbf{k}_{\mu} \uparrow \uparrow \mathbf{k}_{1}\right)$ и противоположно направленных $\left(\mathbf{k}_{\mu} \uparrow \downarrow \mathbf{k}_{1}\right)$ волновых векторах спонтанного и возбуждающего излучений для полуширины центральной линии из (39) имеем:

$$
\begin{gathered}
\Gamma_{\mathrm{ef}}=k_{2}^{2} D, \quad \text { если } \quad \mathbf{k}_{\mu} \uparrow \uparrow \mathbf{k}_{1}, \\
\Gamma_{\mathrm{ef}}=\left(4 k_{1}^{2}+k_{2}^{2}\right) D, \quad \text { если } \quad \mathbf{k}_{\mu} \uparrow \downarrow \mathbf{k}_{1} .
\end{gathered}
$$

Таким образом, при $\mathbf{k}_{\mu} \uparrow \downarrow \mathbf{k}_{1}$ по сравнению со случаем $\mathbf{k}_{\mu} \uparrow \uparrow \mathbf{k}_{1}$ полуширина центральной линии увеличивается в $\left(4 k_{1}^{2}+k_{2}^{2}\right) / k_{2}^{2}$ раз.

На рис. 5 показан спектр резонансной флуоресценции в случае столкновений, сохраняющих фазовую память 
на всех переходах $\left(\widetilde{v}=v=\widetilde{v}_{1}=v_{1}=\widetilde{v}_{2}=v_{2}=\bar{v}\right.$, отсутствие столкновительной релаксации когерентностей $\left.\rho_{m n}, \rho_{k n}, \rho_{m k}\right)$. В этом случае полуширины и амплитуды всех линий спектра анизотропны по отношению к взаимной ориентации волновых векторов спонтанного и возбуждающих излучений. При $\left|G_{2}\right| \gg\left|G_{1}\right|$ для полуширин центральной и боковых линий имеем (при $\left.\bar{v} \gg A_{1}, A_{2}, 2 G\right)$ :

$$
\begin{gathered}
\Gamma_{\mathrm{ef}}=\frac{A_{2}}{2}+\left(2 k_{1}^{2}+k_{2}^{2}-2 \mathbf{k}_{1} \mathbf{k}_{\mu}\right) D, \\
\Gamma_{\mathrm{ef}}^{(1)}=\frac{3}{4} A_{2}+\left(4 k_{1}^{2}+k_{2}^{2}-4 \mathbf{k}_{1} \mathbf{k}_{\mu}\right) \frac{D}{2}, \\
\Gamma_{G}=\frac{A_{1}}{2}+\frac{A_{2}}{4}+\left(2 k_{1}^{2}+k_{2}^{2}-2 \mathbf{k}_{2} \mathbf{k}_{\mu}\right) \frac{D}{2} .
\end{gathered}
$$

Рисунок 5, $а$ иллюстрирует анизотропию полуширин и амплитуд линий спектра по отношению к величине угла $\theta_{1}$ между волновыми векторами $\mathbf{k}_{\mu}$ и $\mathbf{k}_{1}$. При $\left|G_{2}\right| \gg\left|G_{1}\right|$ отношение $A_{G}$ амплитуды каждой из внутренних боковых линий к амплитуде центральной линии равно

$$
A_{G}=\frac{\left(\mathbf{k}_{1}-\mathbf{k}_{2}\right)^{2} v_{T}^{2}}{\bar{v} A_{1}}=\frac{2 D\left(\mathbf{k}_{1}-\mathbf{k}_{2}\right)^{2}}{A_{1}}
$$

и сильно зависит от взаимной ориентации волновых векторов $\mathbf{k}_{1}$ и $\mathbf{k}_{2}$ возбуждающих излучений. На рис. $5, b$ показан спектр резонансной флуоресценции при однонаправленных ( $\mathbf{k}_{1} \uparrow \uparrow \mathbf{k}_{2}$, кривая 1$)$ и противоположно направленных ( $\mathbf{k}_{1} \uparrow \downarrow \mathbf{k}_{2}$, кривая 2$)$ волновых векторах возбуждающих излучений. Обращает на себя внимание тот факт, что при встречном распространении возбуждающих излучений, когда диффузионное уширение проявляет себя в полной мере, интенсивность спонтанного испускания много выше, чем при однонаправленном их распространении. Это связано с тем, что в первом случае уровень $m$ заселяется существенно эффективнее. Проверка по формуле (34) для условий рис. 5 подтверждает данное заключение. Далее, при $\mathbf{k}_{1} \uparrow \downarrow \mathbf{k}_{2}$ по сравнению со случаем $\mathbf{k}_{1} \uparrow \uparrow \mathbf{k}_{2}$ относительная амплитуда $A_{G}$ каждой из внутренних боковых линий увеличивается в $\left(k_{1}+k_{2}\right)^{2} /\left(k_{1}-k_{2}\right)^{2}$ раз, что может стать очень большой величиной при близких частотах переходов. По относительной амплитуде $A_{G}$ внутренних боковых линий можно определять коэффициент диффузии $D$ поглощающих частиц в газе буферных.

Отметим еще одно обстоятельство. Из формул (27) и (37) следует, что при точном резонансе для сильных полей в случае предельно малых значений отношения $\left|G_{1}\right|^{2} /\left|G_{2}\right|^{2}$ (слабая интенсивность возбуждающего поля на переходе $m-n$ и высокая интенсивность возбуждающего поля на смежном переходе $k-n$ ) вероятность спонтанного испускания $w$ крайне мала. Физической причиной малости $w$ является малая населенность возбужденного уровня $m$ из-за того, что в данном случае слабое возбуждающее поле нерезонансно взаимодействует с атомом. Действительно, сильное поле, резонансное переходу $k-n$, расщепляет уровень $n$ на два уровня квазиэнергии, отстоящие от уровня $n$ на $\pm\left|G_{2}\right|$ в частотной шкале. В данной ситуации поле $\left|G_{1}\right|$, резонансное невозмущенному переходу $m-n$, оказывается сильно нерезонансным (на величину $\pm\left|G_{2}\right|$ ) для сформированных переходов. Как следствие, заселенность возбужденного уровня $m$ и интенсивность спонтанного испускания с него становятся крайне малыми.

Представляет естественный интерес осуществить резонансные условия для поля $\left|G_{1}\right|$ при высокой интенсивности поля $\left|G_{2}\right|$. Очевидно, что слабое возбуждающее поле $\left|G_{1}\right|$ будет резонансно взаимодействовать с атомом при отстройках частоты $\Omega_{1}= \pm\left|G_{2}\right|$. При этом интенсивность резонансной флуоресценции должна многократно увеличиться по сравнению со случаем точного резонанса $\Omega_{1}=0$. В обозначенных условиях найдем положения уровней квазиэнергии из характеристического уравнения (7). При $\Omega_{2}=0$ и $\left|G_{1}\right| \rightarrow 0$ из (7) следует: $\alpha_{1}=\Omega_{1}, \alpha_{2,3}= \pm\left|G_{2}\right|$. При $\alpha_{1}=\Omega_{1}=\left|G_{2}\right|$ нижний уровень квазиэнергии оказывается двукратно вырожденным. Учтем далее в уравнении (7) первую поправку на величину $\left|G_{1}\right|^{2}$. В результате получаем, что двукратно вырожденный уровень квазиэнергии расщепляется симметрично на величину $\sqrt{2}\left|G_{1}\right|$, а верхний уровень получает сдвиг, существенно меньший по величине, чем расщепление нижнего. Полная картина уровней квазиэнергий и положений спектральных линий спонтанного испускания строится с помощью выражений (9) для волновых функций. Конкретно в нашем случае спектр спонтанного испускания представлен триплетом, центрированном на частоте возбуждающего излучения $\left|G_{1}\right|$, дублетом, отстроенном в красную сторону на величину $2\left|G_{2}\right|$, и дублетом, отстроенном в синюю сторону на величину $2\left|G_{2}\right|$. Интервал между компонентами дублета и триплета равен $\sqrt{2}\left|G_{1}\right|$. Расчеты спектра спонтанного испускания по точной формуле (формула здесь не приводится ввиду ее громоздкости) для неподвижных и не испытывающих столкновений атомов представлены на рис. 6. Они прекрасно подтверждают приведенные выше соображения. На рис. 6 второй дублет не представлен в силу его очень малой амплитуды. Амплитуды изображенных на этом рисунке линий примерно на 5 порядков больше по сравнению с максимальной амплитудой линии в окрестности частоты $\omega_{m n}$ в случае точного резонанса $\Omega_{1}=0$ и остальных расчетных параметрах тех же, что и для рис. 6.

\section{6. Заключение}

В настоящей работе теоретически исследовано влияние столкновений на спектр резонансной флуоресценции трехуровневых атомов c V-конфигурацией уровней, возбуждаемых двумя монохроматическими полями. Полагалось, что атомы испытывают столкновения с частицами буферного газа. Анализ проведен для случая однородного уширения линий поглощения при достаточно больших давлениях буферного газа, когда частота 
столкновений велика по сравнению с доплеровскими ширинами линий поглощения $\left(\bar{v} \gg k_{1} v_{T}, k_{2} v_{T}\right)$. Задача решена в условиях произвольного изменения (от полного сбоя до полного сохранения) фаз наведенных излучениями дипольных моментов при упругих столкновениях.

Оказалось, что даже при малом доплеровском уширении линий поглощения $\left(k_{1} v_{T}, k_{2} v_{T} \ll \gamma_{1}, \gamma_{2}\right.$, слабоселективное по скоростям взаимодействие атомов с излучением) учет движения атомов совершенно необходим, так как он приводит к резкой трансформации спектра резонансной флуоресценции. Благодаря движению атомов спектр резонансной флуоресценции обладает ярко выраженной анизотропией по отношению к взаимной ориентации волновых векторов спонтанного и возбуждающих излучений. Ширины и амплитуды спектральных компонент оказались зависящими от коэффициента диффузии взаимодействующих с излучением атомов в буферном газе. В рассмотренных условиях несмещенная линия когерентного (рэлеевского) рассеяния в спектре резонансной флуоресценции становится заметной лишь при малых углах $\theta_{1}$ между волновыми векторами $\mathbf{k}_{\mu}$ и $\mathbf{k}_{1}$. Она испытывает только диффузионное уширение (при $\mathbf{k}_{\mu} \neq \mathbf{k}_{1}$ ).

Показано, что ширины линий спектра резонансной флуоресценции сильно зависят от отношения интенсивностей возбуждающих излучений. В этом отношении наиболее интересен спектр спонтанного испускания на переходе $m-n$ в случае столкновений, сохраняющих фазовую память на смежном переходе $k-n$ (отсутствие столкновительной релаксации когерентности $\left.\rho_{k n}\right)$. При $\left|G_{2}\right|<\left|G_{1}\right|$ ширины всех линий спектра по величине сопоставимы с ударной шириной линии поглощения на переходе $m-n$. С ростом интенсивности излучения, резонансного смежному переходу $k-n\left(\right.$ при $\left.\left|G_{2}\right| \gg\left|G_{1}\right|\right)$, происходит резкое сужение центральной и внешних боковых линий, при этом их ширины будут полностью определяться релаксационными константами смежного перехода $k-n$.

В условиях $\Omega_{1}=\Omega_{2}=0$ (точный резонанс возбуждающих излучений с невозмущенными переходами $m-n$ и $k-n)$ при значительном увеличении отношения $\left|G_{2}\right| /\left|G_{1}\right|$ интенсивность спонтанного испускании на переходе $m-n$ резко падает. Это связано с тем, что поглощение излучения на переходе $m-n$ становится сильно не резонансным и, как следствие, слабо заселяется уровень $m$. Сигнал резонансной флуоресценции многократно возрастает при $\Omega_{1}= \pm\left|G_{2}\right|$ (новые условия резонанса в данной конкретной ситуации).

Проведенный в работе анализ показывает, в частности, что на основе исследования спектра резонансной флуоресценции в определенных условиях можно получить полезную информацию о коэффициентах диффузии излучающих атомов в газе буферных частиц.

\section{Финансирование работы}

Исследование выполнено за счет средств субсидии на финансовое обеспечение выполнения государственного задания (проект № АААА-А17-117052210003-4) в Институте автоматики и электрометрии Сибирского отделения РАН.

\section{Конфликт интересов}

Авторы заявляют, что у них нет конфликта интересов.

\section{Список литературы}

[1] Narducci L.M., Scully M.O., Oppo G.-L., Ru P., Tredicce J.R.// Rhys. Rev. A. 1990. V. 42. N 3. P. 1630. doi 10.1103/PhysRevA.42.1630

[2] Narducci L.M., Oppo G.-L., Scully M.O. // Opt. Commun. 1990. V. 75. N 2. P. 111. doi 10.1016/0030-4018(90)90238-O

[3] Zhu Y. // Rhys. Rev. A. 1991. V. 43. N 3. P. 1502. doi 10.1103/PhysRevA.43.1502

[4] Gauthier D.J., Zhu Y., Mossberg T.W. // Phys. Rev. Lett. 1991. V. 66. N 19. P. 2460. doi 10.1103/PhysRevLett.66.2460

[5] Раутиан С.Г., Смирнов Г.И., Шалагин А.М. Нелинейные резонансы в спектрах атомов и молекул. Новосибирск: Наука, 1979. 312 с.

[6] Попов А.К. Введение в нелинейную спектроскопию. Новосибирск: Наука, 1983. 274 с.

[7] Пархоменко А.И., Шалагин А.М. // ЖЭТФ. 2005. Т. 127. № 2. C. 320; Parkhomenko A.I., Shalagin A.M. // JETP. 2005. V. 100. N 2. P. 283. doi $10.1134 / 1.1884669$

[8] Mollow B.R. // Phys. Rev. 1969. V. 188. N 5. P. 1969. doi 10.1103/PhysRev.188.1969

[9] Rautian S.G., Shalagin A.M. Kinetic Problems of Nonlinear Spectroscopy. Amsterdam, NY.: Elsevier Science Publ. Comp., 1991. $439 \mathrm{p}$.

[10] Пархоменко А.И., Шалагин А.М. // ЖЭТФ. 2001. Т. 120. № 4. C. 830; Parkhomenko A.I., Shalagin A.M. // JETP. 2001. V. 93. N 4. P. 723. doi 10.1134/1.1420441

[11] Чепмен C., Каулинг T. Математическая теория неоднородных газов. М.: Изд-во иностр. лит., 1960. 512 с.; Chapman S., Cowling T.G. The Mathematical Theory of Non-Uniform Gases. Cambridge: Cambridge University Press, 1970. $423 \mathrm{p}$.

[12] Пархоменко А.И., Шалагин А.М. // ЖЭТФ. 2008. Т. 133. № 5. C. 984; Parkhomenko A.I., Shalagin A.M. // JETP. 2008. V. 106. N 5. P. 858. doi 10.1134/S1063776108050038 